\title{
Unveiling nanoscale optical and structural properties of TMD monolayers using combined electron spectroscopies
}

Noémie Bonnet ${ }^{1}$, Hae Yeon Lee $^{2}$, Fuhui SHAO ${ }^{3}$, Steffi Woo $^{4}$, Kenji Watanabe ${ }^{5}$, Takashi Taniguchi ${ }^{5}$, Alberto Zobelli $^{1}$, Odile Stéphan ${ }^{6}$, Mathieu Kociak ${ }^{7}$, Jean-Denis Blazit ${ }^{6}$, Silvija Gradecak-Garaj ${ }^{8}$ and Luiz H. G. Tizei ${ }^{9}$

${ }^{1}$ Laboratoire de Physique des Solides/CNRS, Université Paris-Saclay, France, ${ }^{2}$ Massachusetts Institute of Technology, United States, ${ }^{3}$ Université Paris-Saclay, CNRS, Laboratoire de Physique des Solides, United States, ${ }^{4}$ Laboratoire de Physique des Solides/CNRS, Université Paris-Saclay, United States, ${ }^{5}$ National Institute for Material Science, Tsukuba, Japan, ${ }^{6}$ Université Paris-Saclay, CNRS, Laboratoire de Physique des Solides, 91405, Orsay, France, United States, ${ }^{7}$ CNRS, Orsay, Ile-de-France, United States, ${ }^{8}$ National University of Singapore, Singapore, ${ }^{9}$ Université Paris-Saclay, CNRS, Laboratoire de Physique des Solides, 91405, Orsay, France, France

Transition metal dichalcogenide (TMD) semiconductors with the form $\mathrm{MX}_{2}$ ( $\mathrm{M}$ a transition metal, $\mathrm{W}$ or Mo, and $\mathrm{X}$ a chalcogen atom, $\mathrm{S}, \mathrm{Se}$ or $\mathrm{Te}$ ) with the $2 \mathrm{H}$ phase have an indirect bandgap when in bulk, and a direct bandgap when exfoliated down to a monolayer, leading to bright photoluminescence (PL) [1]. These monolayers display attractive properties, such as stable quantum emission [2] or valley-polarization of excitonic transitions [3]. The optical response of these monolayer is determined by excitons, trions, and defected-related transition lines [4].

A major challenge to understanding and optimizing these monolayers, their heterostructures, and possible future devices is the characterization of their optical properties down to the nanometer scale and the correlation of these to their structure and chemistry. Correlated TEM and optical experiments can lead to some understanding of the influence of chemical or structural modifications on optical properties. However, a direct correlation at the nanoscale would be more straightforward. Electron spectroscopy techniques allow for the measurement of optical quantities at high spatial resolution: emission from cathodoluminescence (CL), a nanoscale counterpart of PL [5] and absorption from low-loss electron energy loss spectroscopy (EELS) [6].

In this contribution, the relation between optical, chemical, and structural properties of a h-BN encapsulated monolayer of $\mathrm{WS}_{2}$, with a spatial resolution down to the tens of nanometer scale is discussed. In particular, we have observed spatial variations of the emission of the A exciton $\left(\mathrm{X}_{\mathrm{A}}\right)$ [7], the charged A exciton (trion: $\mathrm{X}^{-}$), and the lower energy localized excitons (L). These spatial variations are linked to chemical changes at the surface of the $\mathrm{WS}_{2}$ monolayers, leading to changes in the $\mathrm{X}_{\mathrm{A}}$ to $\mathrm{X}^{-}$emission intensity ratio.

The experiments were performed in a modified NION Hermes 200 equipped with a monochromator and a Mönch CL system from Attolight [8] (Fig. 1c). CL measurements were done at $60 \mathrm{keV}$, with an electron beam with a 10 mrad convergence semi-angle, and the sample kept at $150 \mathrm{~K}$ using a liquid-nitrogen cooled sample holder. Lowloss EELS was measured with an electron beam with a 10-20 meV energy width and the collection semi-angle of the spectrometer was set to $25 \mathrm{mrad}$. Core-loss EELS and diffraction experiments were done in a NION UltraSTEM 200 , at $100 \mathrm{keV}$, with an electron beam of $30 \mathrm{mrad}$ convergence semi-angle for chemical mapping, and the sample was kept at room temperature.

Fig. 1a represents a scheme of the excitations leading to light emission upon electron-beam excitation of $\mathrm{WS}_{2}: \mathrm{X}_{\mathrm{A}}$, $\mathrm{X}^{-}$, and $\mathrm{L}$ (described before). The $\mathrm{h}-\mathrm{BN}$ encapsulation of the monolayer is crucial to obtain sufficient cathodoluminescence signals [9]. The sample design allows for atomically-resolved imaging, even with the encapsulation (inset of Fig. 1a). In absorption, several excitons $\left(X_{A}, X_{B}\right.$ and $X_{C}$ ) are seen (Fig. 1b). Only the $X_{A}$ is optically active, leading to light emission (a CL spectrum is shown on the inset of Fig. 1b). The energy difference between the absorption and emission of $\mathrm{X}_{\mathrm{A}}$, called Stokes shift (SS), can be locally measured with combined EELS and CL (Fig. 1c). The variations in SS can give insights on the origin of $X_{A}$ energy shifts [10].

Fig. 2 shows an example of the nanometer-scale variations of the $X_{\mathrm{A}}$ and the $\mathrm{X}^{-}$observed in the $\mathrm{h}$-BN encapsulated $\mathrm{WS}_{2}$, as well as the localized, lower energy, L emission lines. The emission (Fig. 2b, c, d) can be directly compared 
to the high angle annular dark field (HAADF) image (Fig. 2a). One sees that the $\mathrm{X}^{-}$emission maxima are related to regions of reduced intensity in the HAADF Images. This HAADF intensity reduction is present in several places of the sample, and displays a similar behavior: the $\mathrm{X}_{\mathrm{A}}$ emission is mostly homogeneous across the field of view, and tends to show a dip in intensity where the $\mathrm{X}^{-}$and/or L emissions are brighter. The $\mathrm{X}^{-}$emission is localized on the darker spots visible in HAADF, while the L lines are very localized in space, but do not match directly the intensity changes observed in the HAADF image.

Hence, from the correlation between nanoscale CL and HAADF imaging, one can already question the origin of the emission variation: $\mathrm{L}$ and $\mathrm{X}^{-}$are not present at the same locations, which points towards possibly different production mechanisms. To understand the nature of the intensity changes seen in HAADF we have used core-loss EELS elemental mapping on the regions of higher intensity. They are rich in $\mathrm{C}, \mathrm{O}$ and $\mathrm{Si}$ [11], which are probably contaminants from the sample preparation. The HAADF intensity variation matches well the chemical map of these contaminants, indicating that the $\mathrm{X}^{-}$localization is probably linked to the absence of the residues, which changes the local dielectric environment. The modification of the local dielectric environment can increase the local density of free electrons, favoring the formation of $\mathrm{X}^{-}$rather than excitons.

In conclusion, the CL/EELS characterization of $\mathrm{WS}_{2}$ encapsulated monolayer together with high-resolution imaging and chemical mapping have shown localization effects of the negatively-charged exciton, $\mathrm{X}^{-}$, that were caused by dielectric environment changes. These variations are not correlated to strain. The observation of localized light emitters paves the way for additional experiments, coupled to light intensity-interferometry, to determine the microscopic structure behind quantum emitters in TMD monolayer $[5,11,12]$.

Acknowledgements: This project has been funded in part by the National Agency for Research under the program of future investment TEMPOS-CHROMATEM (reference no. ANR-10-EQPX-50) and the JCJC program (ANR20-CE42-0020) and from the European Union's Horizon 2020 research and innovation program under grant agreement No 823717 (ESTEEM3) and 101017720 (EBEAM). This work has been supported by Region Île-deFrance in the framework of the DIM SIRTEQ.

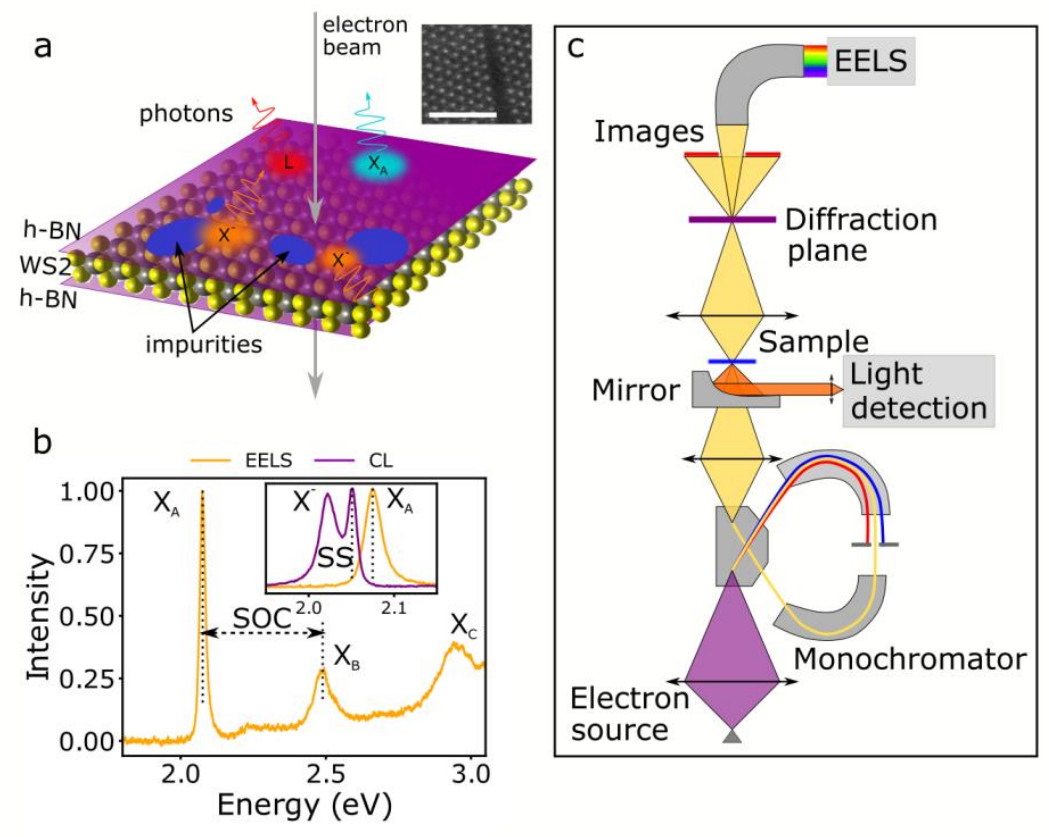

Figure 1. Fig. 1: Scheme of the experiment: (a) Scheme of the WS2 monolayer (W atoms in grey, S atoms in yellow) encapsulated in h-BN (purple). The different types of optical transitions observed in the experiments, XA, $\mathrm{X}$ - and $\mathrm{L}$, when the heterostructure is excited by the electron beam are also sketched. The inset shows an atomically- 
resolved HAADF image of an edge in the encapsulated WS2 monolayer (the scale bar is $2 \mathrm{~nm}$ ). (b) Typical EELS spectrum (orange curve) with the peaks labeled associated to different excitonic transitions excitons (XA, XB, XC). A typical CL emission is shown on the inset (purple curve), where both XA and X-are visible. The energy difference between the absorption (EELS) and emission (CL) of XA is the Stokes shift (SS). (c) Scheme of the STEM microscope used for the experiment. The monochromator allows for high energy resolution low-loss EELS absorption measurements. Light emitted from the sample upon electron excitations (CL) is collected by a parabolic mirror $(\mathrm{NA}=0.5)$ and directed to an optical spectrometer. On the same setup diffraction patterns and images at different collection angles can also be measured in parallel to spectroscopic information. The measurements were performed with the sample kept at $150 \mathrm{~K}$ using a liquid nitrogen cooled sample holder.

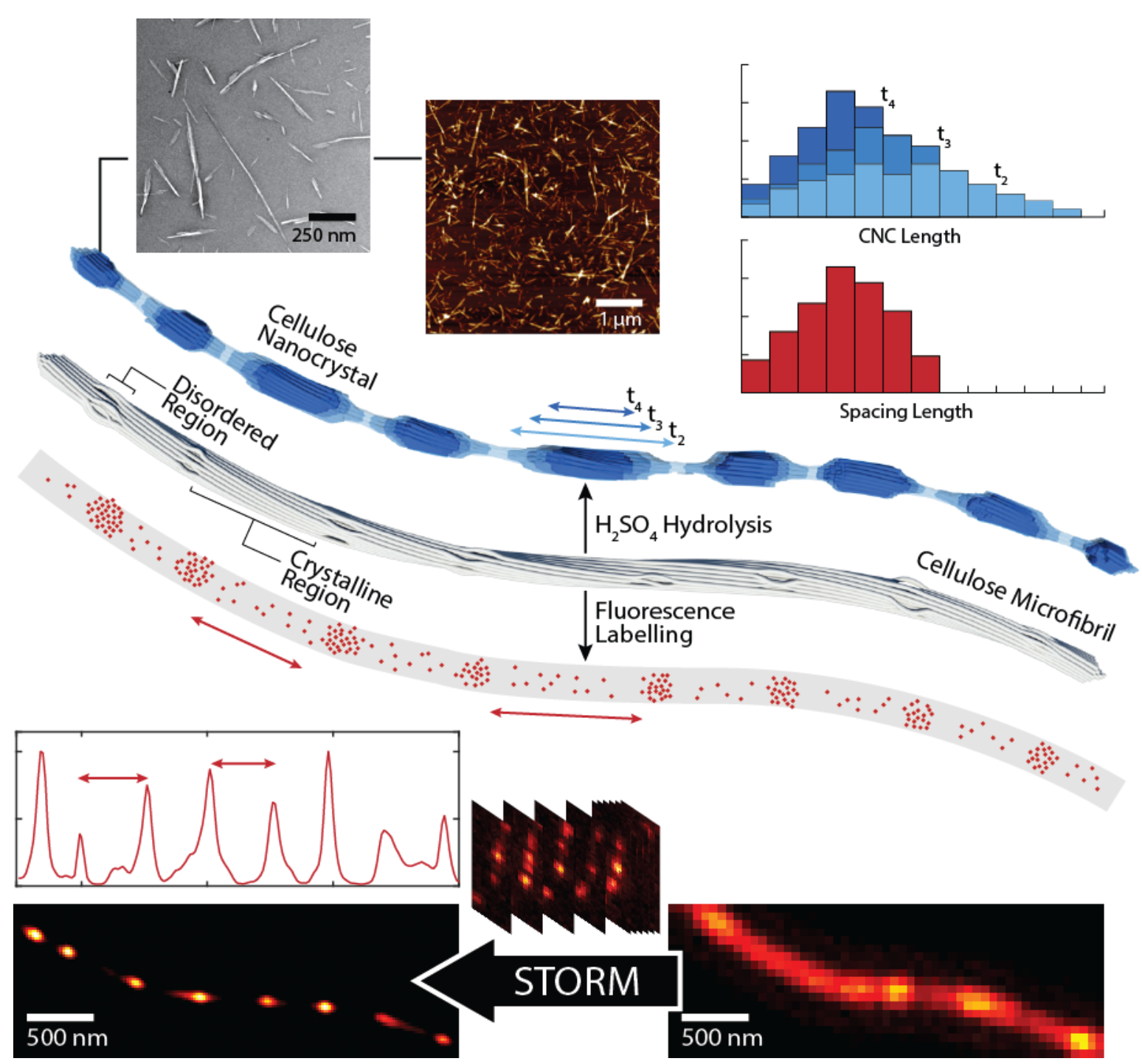

Figure 2. Fig. 2: CL measurements on a heterogeneous area of the WS2 encapsulated monolayer: (a) HAADF image of the area where the CL spectral information was acquired. The brighter areas contain $\mathrm{C}, \mathrm{O}$, and $\mathrm{Si}$, which are probably present due to residues from sample preparation. (b-d) Intensity maps for the X-, XA and L transitions, respectively. The maps were integrated in around the main transitions energies: $2.012 \mathrm{eV}$ for XA, $2.043 \mathrm{eV}$ for X, and $1.997 \mathrm{eV}$ for L. A spatial correlation is observed between intensity maxima of X- map (b) and intensity minima in the HAADF image (a). 


\section{References}

[1] P. V. Kolesnichenko et al, Disentangling the effects of doping, strain and disorder in monolayer WS $\mathrm{W}_{2}$ by optical spectroscopy, 2D Materials, 7, 025008 (2020).

[2] B. Schuler et al, Electrically driven photon emission from individual atomic defects in monolayer $\mathrm{WS}_{2}$, Science Advances, 6,10.1126/sciadv.abb5988 (2020)

[3] Z. Mahfoud et al, Cathodoluminescence in a scanning transmission electron microscope: A nanometer-scale counterpart of photoluminescence for the study of ii-vi quantum dots, J. of Phys. Chem. Lett., 4, 4090 (2013)

[4] A. Arora et al, Dark trions govern the temperature-dependent optical absorption and emission of doped atomically thin semiconductors, Phys. Rev. B, 101, 241413 (2020)

[5] P. Tonndorf et al, Single-photon emission from localized excitons in an atomically thin semiconductor, Optica, 2, 347 (2015).

[6] R. F. Egerton, Electron energy-loss spectroscopy in the electron microscope, Springer, 2011.

[6] X. Xu et al, Spin and pseudospins in layered transition metal dichalcogenides, Nat. Phys.10, 343 (2014).

[7] B. Munkhbat et al, Electrical control of hybrid monolayer tungsten disulfide-plasmonic nanoantenna lightmatter states at cryogenic and room-temperatures, ACS Nano, 14, 1, 1196-1206 (2020)

[8] K. F. Mak et al, Atomically thin MoS2: A new direct-gap semiconductor, Phys. Rev. Lett., 105, 136805 (2010).

[9] S. Zheng et al, Giant enhancement of cathodoluminescence of monolayer transitional metal dichalcogenides semiconductors, Nano Lett.17,6475 (2017).

[10] M. Kociak and L. Zagonel, Cathodoluminescence in the scanning transmission electron microscope, Ultramicroscopy 176, 112 (2017).

[11] N. Bonnet et al, Nanoscale modification of $\mathrm{WS}_{2}$ trion emission by its local electromagnetic environment, arXiv:2102.06140v2 [cond-mat.mes-hall] (2021)

[12] Y. M. He et al, Single quantum emitters in monolayer semiconductors, Nature Nanotech., 10, 497 (2015) 